\title{
Theory of directed transportation of electronic excitation between single molecules through photonic coupling
}

\author{
D. L. Andrews and D. S. Bradshaw \\ Nanostructures and Photomolecular Systems, School of Chemical Sciences, \\ University of East Anglia, Norwich NR4 7TJ, United Kingdom
}

The primary result of UV-Visible photon absorption by complex organic molecules is the population of short-lived electronic excited states. Transportation of their excitation energy between single molecules, formally mediated by near-field interactions, may occur between the initial absorption and eventual fluorescence emission events, commonly on an ultrafast timescale. The routing of energy flow is typically effected by a sequence of pairwise transfer steps over numerous molecules, rather than a single step over the same overall distance. Directionality emerges when there is structure in the molecular organisation. For a chemically heterogeneous system with local order, and with suitable molecular dispositions, automatically unidirectional transfer can be exhibited as the result of a 'spectroscopic gradient'. However it is also possible to exert control over the directionality of excitation flow by the operation of external influences. Examples are the application of an electrical or optical stimulus to the system - achieved by the incorporation of an ancillary polar species, the application of a static electric field or electromagnetic radiation. Most significantly, based on the latter option, an all-optical method has recently been determined that enables excitation transportation to be completely switched on or off, such that the energy flow is subject to controllable photoactivated gating. It is already apparent that this photonic process, termed Optically Controlled Resonance Energy Transfer, has potentially numerous applications. For example, it represents a new basis for optical transistor action.

Keywords: Ultrafast photonics, energy transportation, electronic excitation, optical transistor, photophysics, organic molecules, near-field, light manipulation, photoactivation

\section{INTRODUCTION}

The absorption of UV-Visible light by a heterogeneous organic material will generally result in individual molecules or chromophores being promoted to short-lived electronic excited states. Single- or multi-step intermolecular transfer of the excitation may then occur, following the initial absorption, and prior to an eventual re-emission or dissipative loss. Each transfer event commonly occurs on an ultrafast timescale. The overall routing of energy flow through the material is determined by the sequence of these transfer steps, with the initial and destination chromophores differing either chemically or through local modifications of energy level structure arising from differences in electronic environment. In the near-field, each elementary transfer step takes the form of a radiationless pairwise interaction, generally occurring between an electronically excited donor and an electronically distinct acceptor initially residing in its ground state. Förster ${ }^{1}$ first demonstrated the Coulombic origin of this interaction, now commonly known as resonance energy transfer (RET), also deriving its inverse sixth power dependence on the donor-acceptor separation. It has since been shown ${ }^{2-5}$ that the Förster interaction is the short-range limit of a unified transfer mechanism whose modified rate equation, including terms with inverse fourth power and inverse square dependences on separation, is valid over all distances. In an optically dense system containing a large number of chromophores, many of these pairwise interactions will typically occur before the system enters into a stable state, and the energy transfer path accordingly features a series of short hops rather than a single long one - largely as a result of the dominant term having an inverse sixth power distance dependence. As such, multi-step resonance energy transfer might be expected to have the character of a random walk as does indeed occur in homogeneous single-component systems. However, with suitable chromophore differentiation, functionally unidirectional transfer is exhibited in multichromophore systems, assisted by suitable chromophore disposition, and often the operation of a spectroscopic gradient. ${ }^{6}$ The latter terms signifies that a small amount of energy is commonly lost through vibrational relaxation after each transfer step, the species acting as the next acceptor receiving energy associated with a longer optical wavelength than the donor initially acquired. This causes back-transfer to be very inefficient, due to the poorer overlap of the acceptor emission and donor absorption spectra. 
Directional RET is an extremely significant feature in determining the mode of operation and the efficiency of both photosynthetic and biomimetic light-harvesting units. The light-harvesting systems of purple bacteria in particular have been extensively studied and characterized, and the emulation of their high efficiency is a key goal in the development of new energy harvesting materials. ${ }^{7-10}$ In order to most effectively utilize the sunlight that falls on them, photosynthetic organisms have a system of antenna complexes surrounding the reaction centres where photosynthesis takes place. The complexes absorb sunlight and the acquired energy migrates towards the reaction centre by a series of short-range, radiationless energy transfer steps. In the overall migration of energy from the site of its initial deposition to the site of its chemical action, the spectroscopic gradient is one of the key directional principles obviating random diffusion; by such means, energy absorbed by outlying complexes is quickly and efficiently directed towards the reaction centre - a process often referred to as channelling or funnelling. Not only does this allow an organism to harvest light incident on a large surface area, but by pooling energy from a large number of antenna chromophores, energy of a higher equivalent frequency can be produced. This is essential, since the majority of the incident light from the sun has too low a frequency for its individual photons to effect photosynthesis.

It is not only spectroscopic properties of the chromophores that determine the direction of energy flow; the chromophore positioning and orientation are also important. Two-dimensional optical spectroscopy can unveil the intricate interplay between spectral and spatial overlap features in light-harvesting complexes, as beautifully exhibited in recent studies on the Fenna-Olsen-Matthews bacteriochlorophyll $a$ protein of green sulphur bacteria. ${ }^{11}$ Interrogating the system with a sequence of ultrashort laser pulses, the optical response of the sample can be interpreted to reveal linear absorption processes as well as couplings between chromophores, and dynamical aspects of the energy transfer. The results show that excitation relocation does not simply proceed by stepwise transfer from one energy state to another of nearest energy - it depends on strong coupling between chromophores, determined by the extent of their spatial overlap. Thus, excitation relocation may involve fewer intermediary chromophores than might otherwise be expected.

The efficiency of photosynthetic units has encouraged the design of a variety of synthetic light-harvesting systems that can mimic their energy funnelling properties. The materials which have received most attention are dendrimers organic macromolecules consisting of chromophore units repeatedly branching out from a central core designed to act as an excitation trap. ${ }^{12,13}$ The branching functionalization of terminal groups leads to successive generations of structures, each with an increased number of peripheral antenna chromophores. In ideal cases the requisite spectroscopic gradient is established through chemically similar chromophores in generationally different locations having different electronic properties, due to subtly differing chemical environments. This arrangement expedites an emulation of the energy funnelling observed in natural light-harvesting systems: peripheral chromophores absorb the incident photons, excitation energy relocates across the structure, ${ }^{14,15}$ and is ultimately funnelled into the core. ${ }^{16}$ Striking examples of this principle can be seen in early work on perylene-functionalised phenylacetylene dendrimers. ${ }^{17,18}$ More recent work on dendrimers has seen a number of variations on the basic dendrimeric structure, mostly with branching motifs of threefold and fourfold local symmetry based on $(1,3,5)$-substituted benzene and porphyrin rings, respectively. ${ }^{19-22}$

Whilst the spectral and geometric principles that govern the directionality of energy flow in multi-chromophore systems are largely understood, recent developments have identified new possibilities for effecting externally determined directional control. In particular it has emerged that, by a variety of means, it is possible to exploit optical and electronic perturbations of light-harvesting systems that exert significant additional directing influences on the energy transit. The delineation and analysis of these mechanisms is the main subject of this paper. In Section 2 the general theory underlying such influences is reviewed. One particularly attractive mechanism allowing greater control of the energy transfer is Optically Controlled Resonance Energy Transfer (OCRET) - a process that, through the input of non-resonant throughput radiation, more efficiently manipulates the flow of excitation energy. By such means it is shown that all-optical switching becomes possible - this process is detailed in Section 3. Future prospects for the utilization of directional control are discussed in the concluding Section 4.

\section{INFLUENCES ON ENERGY TRANSFER}

To understand and to correctly model the multi-step energy transfer mechanism that typifies the operation of many photoactive organic complexes, an appropriate starting point is the prototypical case of energy transfer from an excited donor to a neighbouring acceptor in its ground state. In this section, following a brief outline of the fundamental theory

6999-12 V. 1 (p.2 of 10) / Color: No / Format: A4 / Date: 3/7/2008 7:29:45 AM

SPIE USE: DB Check, Prod Check, Notes: 
for the donor-acceptor pair, consideration is given to the influences of other neighbouring chromophores, and to the effects of applying either a static electric field or electromagnetic radiation. A simple depiction of the four processes to be covered is given in Figure 1.

\subsection{Fundamental Theory}

Each transfer of energy between a donor chromophore, $A$, and an acceptor, $B$, may be represented by the chemical equation;

$$
A^{*}+B \longrightarrow A+B^{*}
$$

Here, neither the mechanism for the excitation of $A$ nor the subsequent decay of $B$ need be included, on the grounds that they are kinetically separable events. Assuming a donor-acceptor separation corresponding to the near-field region (i.e., a distance small compared to the optical wavelength for the energy transferred), then by use of the Fermi Rule the rate of energy transfer can be represented as; ${ }^{23}$

$$
W_{A B}=\frac{9 c^{4} \kappa_{A B}^{2}}{8 \pi \tau_{A} R_{A B}^{6}} \int F_{A}(\omega) \sigma_{B}(\omega) \omega^{-4} \mathrm{~d} \omega
$$

where $F_{A}(\omega)$ is the donor emission spectrum, $\sigma_{B}(\omega)$ denotes the absorption cross-section of the acceptor and $\hbar \omega$ is the energy transferred - the integration being performed over a frequency range that is specifically limited to the salient donor emission and acceptor absorption regions. Also in equation (1), $\tau_{D}$ is the donor radiative lifetime (the product of the measured fluorescence lifetime and quantum yield), $\mathbf{R}_{A B}=\mathbf{R}_{A}-\mathbf{R}_{B}$ represents the vector separation of the chromophores, and the orientation factor is defined as;

$$
\kappa_{A B}=\left(\hat{\boldsymbol{\mu}}^{A} \cdot \hat{\boldsymbol{\mu}}^{B}\right)-3\left(\hat{\boldsymbol{\mu}}^{A} \cdot \hat{\mathbf{R}}_{A B}\right)\left(\hat{\boldsymbol{\mu}}^{B} \cdot \hat{\mathbf{R}}_{A B}\right)
$$

with $\hat{\boldsymbol{\mu}}^{A}, \hat{\boldsymbol{\mu}}^{B}, \hat{\mathbf{R}}_{A B}$ as unit vectors in the direction of the dipole transition moments and the donor-acceptor separation vector, respectively. The $\kappa$ factor embodies another important facet of the energy transfer, an orientational dependence determined by the chromophore architecture. For certain orientations of the displacement vector and dipole transition moments - for example if all three are mutually orthogonal - the $\kappa$ factor will vanish, resulting in zero energy transfer. At the other extreme, optimization of the energy transfer rate is achieved by either transition moments being parallel or anti-parallel to the separation vector. ${ }^{24}$ Further analysis of equation (1) indicates that energy transfer is also strongly influenced by the separation and spectral features of the two chromophores. In detail, the efficiency of transfer depends on; the extent of overlap between the emission spectrum of the donor and the absorption spectrum of the acceptor, the donor-acceptor geometry, and most importantly the $R^{-6}$ distance dependence.

\subsection{Static Electric Field}

If a donor-acceptor pair of chromophores is placed in a static electric field, the rate of energy transfer between them will be modified by their interaction with the electric field. ${ }^{25}$ It transpires that the mechanism for delivery of energy to the acceptor then comprises four pathways, to be understood as follows. The quantum amplitude for the overall process is dominated by the usual field-independent term, but also comprises correction terms - the most significant of which entail linear coupling of the static field with either $A$ or $B$, and another of which involves coupling of the static field at both $A$ and $B$. From the Fermi Rule, the rate of energy transfer is in turn determined from the square of the quantum amplitude. Figure 2 exhibits a graph showing a typical form for a variation in the rate of energy transfer with the orientation of the static electric field displacement vector, based on typical values for the static field strength and transition dipole moments. When the static field engages with a transition, it is associated with different selection rules - which can be formally identified with those of a two-quantum transition. The amenability of the static field-induced mechanism to directing energy transfer is primarily based on the case of chromophores exhibiting a (single-photon) dipole-forbidden but two-quantum allowed transition. Energy transfer does not occur to or from such a species without the presence of a static field, allowing a switchable electric field to control the delivery of energy to the acceptor. 


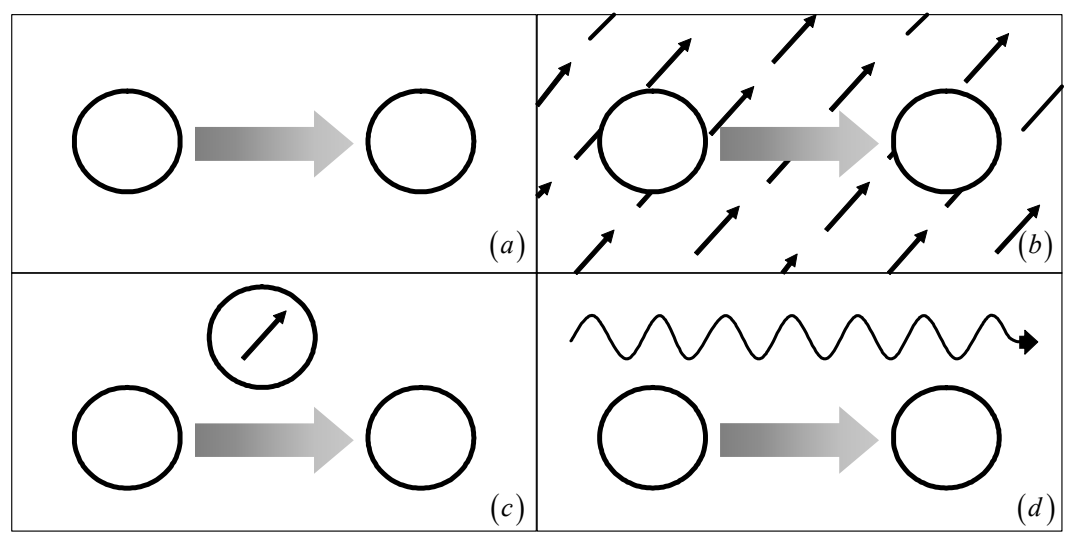

Figure 1. Depictions of: $(a)$ resonance energy transfer; $(b)$ electric field induced energy transfer; $(c)$ third-body energy transfer; $(d)$ laser-assisted resonance energy transfer. In each case, energy transfers from the donor on the left to the acceptor on the right; in the latter three cases an auxiliary influence affects the rate of transfer.

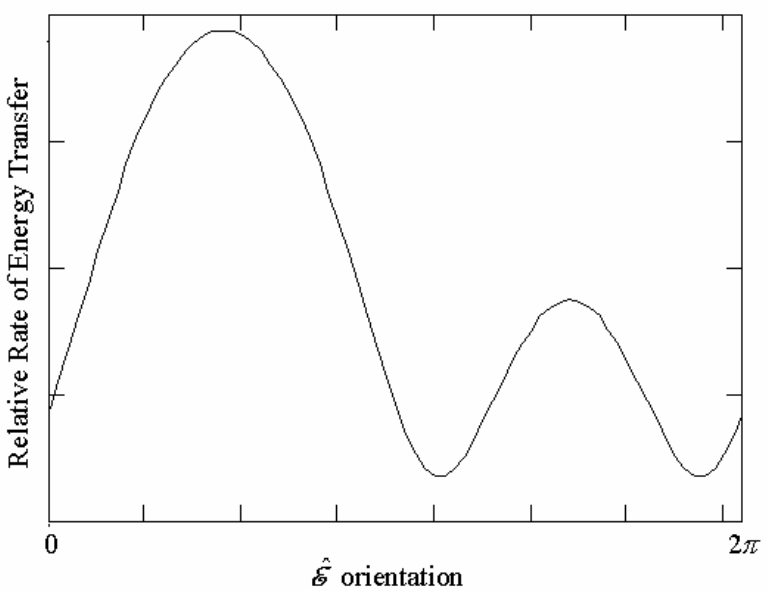

Figure 2. Influence of a static field on energy transfer as the polarization vector of the static field is rotated relative to the transfer pair. Here, $\hat{\mathcal{E}}$ is the electric displacement vector of the static field, the donor and acceptor transition moments are parallel and equal in magnitude $\left(10^{-29} \mathrm{C} \mathrm{m}\right)$ and the static field strength is $10^{10} \mathrm{~V} \mathrm{~m}^{-1}$.

\subsection{Neighbouring Dipole}

In conventional theory, RET is generally described in terms of a coupling between electronically isolated chromophores. However, in reality most systems comprise a large number of chromophores, and each transfer event takes place in an electronic environment that may be significantly modified by one or more chromophores in proximity to the interaction pair. If such chromophores (non-participants in terms of the energy transfer routing) are polar, then it can be expected that the static fields they locally produce will exert an influence over nearby energy transfer events. In this connection it is possible for a variety of species to play the role of a 'third body' $M$. For example, in a dendrimeric system the latter might be another chromophore of the same type as either donor or acceptor, within the superstructure and in the same general vicinity. In a photosynthetic complex the third body might be an ancillary pigment, or even one residue of a support protein unit. The possible involvement of such species in exerting an influence on the rate of donor-acceptor 
transfer has received surprisingly little attention, yet it transpires that surrounding chromophores, especially any that are strongly polar, can substantially affect the rate of energy transfer without themselves changing state. This mechanism can be represented in general terms by;

$$
A^{*}+B+M \longrightarrow A+B^{*}+M
$$

Associated with this mechanism, in addition to the usual quantum amplitude for direct transfer not explicitly involving the chromophore $M$, as described in the initial section, three further distinct contributions to the amplitude can be identified: a static dipole of $M$ interacts with either $A$ or $B$, or a dynamic dipole of $M$ acts as an intermediary for the energy transfer. In a sense the static interaction is comparable to that described in the last sub-section, except that here the field is produced in situ rather than being externally delivered.

Previous research into such an influence on energy transfer ${ }^{26}$ has shown that the third body chromophore can have a very significant effect on the rate of energy transfer when that chromophore is close to either one of the donor-acceptor pair, typically at distances less than $1.5 \mathrm{~nm}$. In this region, the third-body influence typically ranges from a $50 \%$ enhancement of the rate to a $40 \%$ reduction, depending on the precise arrangement of the chromophores involved. Although the exact rate distribution with respect to position depends on the relative dipole moment orientation of the three chromophores, in general the influence of $M$ is greatest when the dipole is positioned just outside the region of wavefunction overlap with $A$ or $B$. An example of this behaviour is given in Figure 3, and a possible experimental observation of the effect is discussed in ref. 27. It is imperative to take this mechanism into account when analyzing systems with a large number of closely spaced chromophores, such as dendrimers and other light-harvesting materials.

\subsection{Electromagnetic field}

The rate of pairwise transfer may also be modified by an intense, pulsed throughput laser beam. This involves the radiation being forward Rayleigh-scattered, cooperatively by the donor-acceptor pair (Figure 4) - effectively leaving the throughput beam unchanged, but either enhancing or diminishing the efficiency of energy transfer between the two chromophores. In the former, more interesting case the process is known as laser-assisted resonance energy transfer (LARET). ${ }^{28}$ In broad terms, this laser-modified mechanism for energy transfer bears some similarity to the static field-induced case described in section 2.2, except that here a dynamic optical field is applied. Surprisingly, static dipoles of the donor and acceptor still play a role, despite the oscillatory character of the field - but those which enter the rate expression now relate to electronically excited states. In other words, this mechanism operates through shifts in the electron distributions, associated with the donor decay and the acceptor excitation transitions

Detailed analysis of LARET has shown that the standard RET pathway is dominant at incident intensities below about $10^{13} \mathrm{~W} \mathrm{~m}^{-2}$. At intensities of around $10^{16} \mathrm{~W} \mathrm{~m}^{-2}$ (relatively high, but still easily produced using pulsed lasers) an enhancement to the rate of $10 \%$ or more can be observed for non-polar systems. However for chromophores which are both polar and chiral, additional terms arise, and these can lead to rate enhancements as high as $30 \%$ or more. Due to the linear and quadratic dependencies on irradiance for the LARET rate contributions, any increase in the intensity of the throughput laser light can effect a disproportionately greater enhancement to the rate, although the availability of higher intensities (and the possibility of introducing competing processes such as multiphoton dissociation) must then be taken into account. Using off-resonant laser light to augment energy transfer should find many applications in artificial systems, since it provides an easily controlled, switchable process that nonetheless has a passive role with regards to the system. We explore this in greater detail in the following section.

\section{OPTICAL CONTROL OF ENERGY TRANSFER}

In the previous section, a variety of means for effecting directed energy transfer have been discussed. Each mechanism offers distinct opportunities for future applications, which are being brought closer to fruition as new photonic technology is developed. One of the operational principles under consideration for new devices is to configure a resonant coupling of the energy of throughput optical radiation in matter with a suitable absorption profile, enabling excitation to propagate between nearby molecules having a suitably matching frequency response. Many of the proposed 


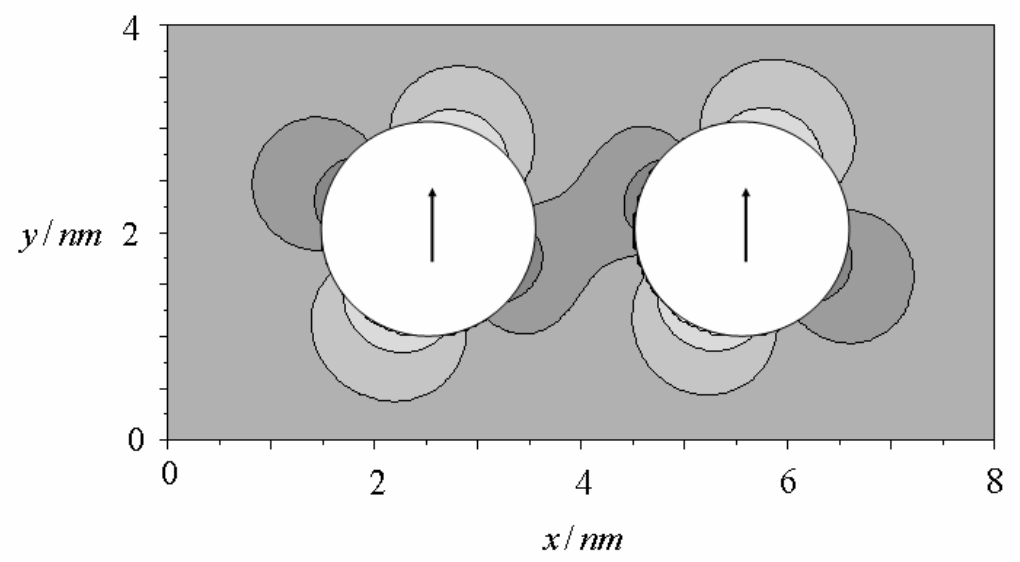

Figure 3. Influence of neighbouring dipole position on the rate of energy transfer between two chromophores. Both chromophore transition moments are aligned parallel to the $y$-axis, the ancillary dipole at an angle of $\pi / 4$ to the $y$-axis. The influence on the RET rate increases with the shade of gray, the darkest sections representing a 200\% increase of the rate over the lightest area. White areas denote positions of significant wavefunction overlap, where a different (exchange) mechanism would come into play.

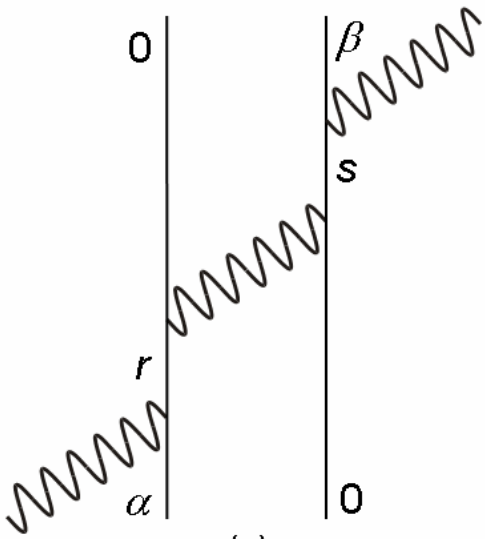

(a)

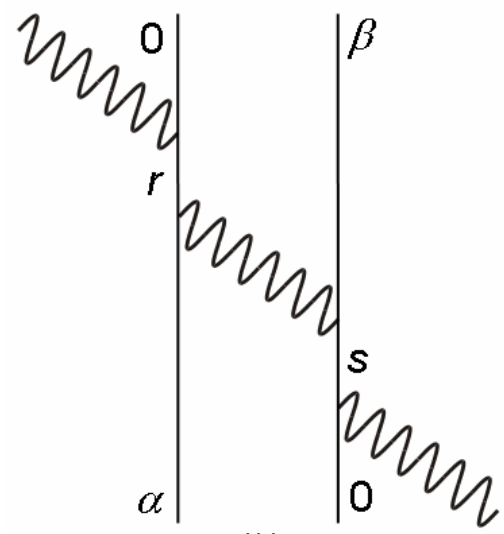

(b)

Figure 4. Feynman diagrams for OCRET. Here, $|0\rangle$ represents a molecule in the ground state; $|\alpha\rangle$ and $|\beta\rangle$ relate to the excited state of the donor (on the left) and acceptor (right), respectively, with $|r\rangle$ and $|s\rangle$ as the corresponding intermediate states. In detail, diagram (a) depicts an instantaneous mechanism involving photon absorption and emission at the donor and acceptor, respectively, with a coupling photon created at the donor and annihilated at the acceptor; thus excitation is transferred from $A$ to $B$.

Diagram $(b)$ is a permutation that will achieve an identical final result.

systems thus involve internal transfers of optical excitation energy, the RET activated by an applied electric ${ }^{29,30}$ or optical field $^{31-35}$ for example. Such devices hold significant promise for the furtherance of ultrafast communication and signal processing systems. Within this context, and as one example of directed energy transfer, we now explore a nanoscale optical switching concept based on LARET. Although electrical field influences are also capable of activating forbidden energy transfer, and thus forming the basis for such an application, an adapted LARET process proves to be the most amenable to controlled nanoscale implementation.

By employment of a local geometric configuration in which $\hat{\boldsymbol{\mu}}^{A}, \hat{\boldsymbol{\mu}}^{B}, \hat{\mathbf{R}}_{A B}$ are mutually orthogonal, as stated earlier, the $\kappa$ factor will vanish and no RET will occur. However, the process returns on application of an off-resonance 
input beam, in a process termed OCRET - a specific instance of LARET determined by this geometric condition. Therefore, OCRET represents an all-optical switching mechanism in which energy transfer is activated and deactivated with the laser beam turned 'on' and 'off', respectively. To specifically determine the rate of energy transfer for the OCRET mechanism, a time-dependent perturbation method is required. In the near-field, the quantum amplitude arising from the input auxiliary beam is given by;

$$
M_{f i}^{(4)}=\left(\frac{n \hbar c k}{8 \pi \varepsilon_{0}^{2} V R^{3}}\right) e_{i} \bar{e}_{l}\left(\delta_{j k}-3 \hat{R}_{j} \hat{R}_{k}\right)\left(S_{i j}^{A}(k) S_{l k}^{B}(-k)+S_{i j}^{B}(k) S_{l k}^{A}(-k)\right)
$$

where $n$ is the number of photons (proportional to intensity) in the quantization volume $V$, and the implied summation convention for repeated Cartesian tensor indices is employed. Furthermore, $\mathbf{e}$ and $\hbar c k$ denote the polarization vector (an overbar denoting complex conjugation) and energy of the input photon, respectively. Also in (3) is the generalized polarizability, explicitly given by;

$$
S_{i j}^{\xi}( \pm k)=\sum_{r}\left\{\frac{\mu_{i}^{f r} \mu_{j}^{r i}}{E_{r f} \pm \hbar c k}+\frac{\mu_{j}^{f r} \mu_{i}^{r i}}{E_{r i} \mp \hbar c k}\right\},
$$

where the transition dipole moments are designated by the shorthand notation $\mu^{x y}=\langle x|\mu| y\rangle$, and energy differences are expressed in the form $E_{x y}=E_{x}-E_{y}$. Further, $f$ signifies the final electronic state of molecule $\xi, i$ is the initial state, and $r, s$ are intermediates. To a good approximation, it can be assumed that the sums of equation (4) are limited to the three states that determine the most prominent optical features. These are denoted $|0\rangle,|\sigma\rangle,|\alpha\rangle$ for donor $A$, and $|0\rangle,|\tau\rangle$, $|\beta\rangle$ for acceptor $B$ - where $|\alpha\rangle$ and $|\beta\rangle$ are the levels between which energy transfer occurs. It is also expedient to select a frequency for the input radiation that has a resonance offset with respect to the positioning of these levels, a condition expressible as $E_{\sigma \alpha}=\hbar c k+\Delta E_{A}$, where $\Delta E_{A}$ is a non-zero energy with magnitude significantly lower than a typical transition energy. An expression of similar form, $E_{\tau \beta}=\hbar c k+\Delta E_{B}$, is assumed for $B$. The outcome of applying these conditions is that one of the summands that contribute to equation (3) is significantly larger in magnitude than the rest. Hence, (3) becomes;

$$
M_{f i}^{(4)}=\left(\frac{n \hbar c k\left(\mathbf{e} \cdot \boldsymbol{\mu}^{\sigma \alpha}\right)\left(\overline{\mathbf{e}} \cdot \boldsymbol{\mu}^{\beta \tau}\right)}{8 \pi \varepsilon_{0}^{2} V R^{3} \Delta E_{A} \Delta E_{B}}\right)\left(\boldsymbol{\mu}^{0 \sigma} \cdot \boldsymbol{\mu}^{\tau 0}-3\left(\boldsymbol{\mu}^{0 \sigma} \cdot \hat{\mathbf{R}}\right)\left(\boldsymbol{\mu}^{\tau 0} \cdot \hat{\mathbf{R}}\right)\right)
$$

Next, to proceed to the geometric conditions, we introduce a Cartesian basis in which the donor-acceptor displacement vector $\mathbf{R}$ is identified with the $z$-direction. The vectors $\hat{\mu}_{A} \equiv \hat{\boldsymbol{\mu}}^{0 \alpha}$ and $\hat{\boldsymbol{\mu}}_{B} \equiv \hat{\boldsymbol{\mu}}^{\beta 0}$ are chosen unambiguously as being directed in the $\hat{\mathbf{i}}$ and $\hat{\mathbf{j}}$ directions respectively and, by a judicious choice which is readily justifiable on symmetry grounds, $\hat{\mu}^{0 \sigma}=\hat{\mathbf{k}}$ and $\hat{\boldsymbol{\mu}}^{\sigma \alpha}=\hat{\mathbf{j}}$. Due to the mutual orthogonality in the triad of vectors that determines the conditions for OCRET, the dipole orientations of the acceptor are determined by taking these vectors and rotating each by $90^{\circ}$ around the $z$-axis, so that $\hat{\boldsymbol{\mu}}^{\tau 0}=\hat{\mathbf{k}}$ and $\hat{\boldsymbol{\mu}}^{\beta \tau}=-\hat{\mathbf{i}}$. For convenience $A$ and $B$ are chosen to belong to identical symmetry groups, although the same calculational method allows application to systems where this is not necessarily the case. Therefore, employing this configuration, equation (5) becomes;

$$
M_{f i}^{(4)}=\left(\frac{-n \hbar c k|\mu|^{4} \kappa^{\prime}}{8 \pi \varepsilon_{0}^{2} V r^{3} \Delta E_{A} \Delta E_{B}}\right) \sin ^{2} \phi \cos \theta \sin \theta,
$$

where the orientation factor is $\kappa^{\prime}=\left(R^{2}-3 r^{2}\right) / R^{2}=-2$, given that $\mathbf{R}=r \hat{\mathbf{k}}, r$ being the displacement of $B$ from $A$. Furthermore, the given angles denote the orientation of $\mathbf{e}$ in spherical coordinates (with the input photon polarization taken as linear). Once again, for simplicity all transition dipole moments are assumed to have similar magnitude - 
although the general result also accommodates other cases. Equation (6) delivers a non-zero result, unlike the now precluded RET. The rate of energy transfer, secured from the Fermi Golden Rule, is given by;

$$
W_{A B}=\frac{4 K C^{2} I^{2}}{r^{6}} \sin ^{4} \phi \cos ^{2} \theta \sin ^{2} \theta
$$

where the parameters are defined as $K=|\mu|^{4} \rho_{f} / 8 \pi \varepsilon_{0}^{2} \hbar$ (in which $\rho_{f}$ is the density of states), $C=|\mu|^{2} / 2 c \varepsilon_{0} \Delta E_{A} \Delta E_{B}$ and $I \equiv n \hbar c^{2} k / V$ is the irradiance. It is noteworthy that with $\phi=0^{\circ}, \theta=0^{\circ}$ or $\theta=90^{\circ}$, no energy transfer to the acceptor occurs. Also, the familiar $R^{-6}$ separation dependence is again apparent.

\section{FUTURE APPLICATIONS}

The processes presented in this paper represent prospects for a number of different ways in which a vectorial character can be produced or enhanced in multi-step resonance energy transfer. Each mechanism is particularly amenable to nanophotonic device implementation, since the propagation of electronic energy by RET is specifically directed over a sub-wavelength scale. Although the mechanisms for controlling energy flow are only just beginning to receive due attention, it is already clear that their detailed methods of implementation will differ, according to the mechanism to be deployed.

Exercising electric field control over RET appears to have potential for applications in the optimization of organic light-harvesting materials. To operate effectively, the mechanism requires levels of field that would be most easily sustained within microscopic or sub-microscopic domains (obviating the extremely high voltages otherwise required), and these may be best achieved through surface field effects - for example, deriving from a nearby silver particle. ${ }^{36,37}$ The capacity to switch a directing field 'on' and 'off' offers intriguing possibilities, though one that would necessarily be limited by the speed of the associated electronics. To affect such control more directly at the molecular level, utilizing local fields generated by neighbouring dipoles, allows the necessary fields to be produced more readily - but such a system is less amenable to real-time experimental control. Here one might envisage composite materials, whose molecular architecture is designed to deliver optically acquired energy from antenna chromophores to suitable traps, expedited by the incorporation of strongly polar groups.

The final mechanism discussed above and elaborated in Section 3 - the case of optically induced RET switching appears to be the most promising for device implementation. It is highly significant that the laser systems capable of delivering the necessary levels of irradiance are precisely those that also offer directly controllable, ultrafast speeds of switching. A workable all-optical switching device will probably involve an array system; an analysis of which has recently been published. ${ }^{38-40}$ Ongoing research will explore the obvious switching, logic gate and transistor attributes of OCRET, whilst the aspect that might hold the greatest promise for optical communications and data transmission is the capacity of such a system to act as an optical buffer.

\section{ACKNOWLEDGEMENTS}

We wish to thank the Leverhulme Trust for providing the financial support for this research.

\section{REFERENCES}

1. T. Förster, "Zwischenmolekulare Energiewanderung Und Fluoreszenz”, Ann. Phys. 2, pp. 55-75., 1948.

2. G. Juzeliūnas and D. L. Andrews, "Quantum electrodynamics of resonance energy transfer", Adv. Chem. Phys. 112 pp. 357-410, 2000.

3. G. J. Daniels, R. D. Jenkins, D. S. Bradshaw and D. L. Andrews, "Resonance energy transfer: The unified theory revisited”, J. Chem. Phys. 119, pp. 2264-2274, 2003.

4. A. Salam, "A general formula for the rate of resonant transfer of energy between two electric multipole moments of arbitrary order using molecular quantum electrodynamics", J. Chem. Phys. 122, 044112, 2005. 
5. A. Salam, "Resonant transfer of excitation between two molecules using Maxwell fields", J. Chem. Phys. 122, $044113,2005$.

6. D. L. Andrews and J. Rodríguez, "Resonance energy transfer: Spectral overlap, efficiency, and direction”, J. Chem. Phys. 127, 084509, 2007.

7. V. Sundström, T. Pullerits and R. van Grondelle, "Photosynthetic light-harvesting: Reconciling dynamics and structure of purple bacterial LH2 reveals function of photosynthetic unit", J. Phys. Chem. B 103, pp. 2327-2346, 1999.

8. B. P. Krueger, G. D. Scholes, I. R. Gould and G. R. Fleming, "Carotenoid mediated B800-B850 coupling in LH2", Phys. Chem. Comm. 8, pp. 34-40, 1999.

9. A. W. Roszak, T. D. Howard, J. Southall, A. T. Gardiner, C. J. Law, N. W. Isaacs and R. J. Cogdell, "Crystal Structure of the RC-LH1 Core Complex from Rhodopseudomonas palustris", Science 302, pp. 1969-1972, 2003.

10. Z. Katiliene, E. Katilius, G. H. Uyeda, J. C. Williams and N. W. Woodbury, "Increasing the rate of energy transfer between the LH1 antenna and the reaction center in the photosynthetic bacterium Rhodobacter sphaeroides", J. Phys. Chem. B 108, pp. 3863-3870, 2004.

11. T. Brixner, J. Stenger, H. M. Vaswani, M. Cho, R. E. Blankenship and G. R. Fleming, "Two-dimensional spectroscopy of electronic couplings in photosynthesis", Nature 434, pp. 625-628, 2005.

12. A. Bar-Haim and J. Klafter, "Dendrimers as light harvesting antennae", J. Lumin. 76-7, pp. 197-200,1998.

13. A. Bar-Haim and J. Klafter, "Geometric versus energetic competition in light harvesting by dendrimers", J. Phys. Chem. B 102, pp. 1662-1664, 1998.

14. S. Tretiak, V. Chernyak and S. Mukamel, "Localized electronic excitations in phenylacetylene dendrimers", J. Phys. Chem. B 102, pp. 3310-3315, 1998.

15. S. F. Swallen, Z. Y. Shi, W. H. Tan, Z. F. Xu, J. S. Moore and R. Kopelman, "Exciton localization hierarchy and directed energy transfer in conjugated linear aromatic chains and dendrimeric supermolecules", J. Lumin. 76-7 pp. 193-196, 1998.

16. A. Adronov and J. M. J. Frechet, "Light-harvesting dendrimers", Chem. Commun., pp. 1701-1710, 2000.

17. C. Devadoss, P. Bharathi and J. S. Moore, "Energy transfer in dendritic macromolecules: Molecular size effects and the role of an energy gradient", J. Am. Chem. Soc. 118, pp. 9635-9644, 1996.

18. M. R. Shortreed, S. F. Swallen, Z. Y. Shi, W. H. Tan, Z. F. Xu, C. Devadoss, J. S. Moore and R. Kopelman, "Directed energy transfer funnels in dendrimeric antenna supermolecules", J. Phys. Chem. B 101, pp. 6318-6322, 1997.

19. U. Hahn, M. Gorka, F. Vögtle, V. Vicinelli, P. Ceroni, M. Maestri and V. Balzani, "Light-harvesting dendrimers: Efficient intra- and intermolecular energy-transfer processes in a species containing 65 chromophoric groups of four different types", Angew. Chem. Int. Ed. 41, pp. 3595-3598, 2002.

20. F. Würthner and A. Sautter, "Energy transfer in multichromophoric self-assembled molecular squares", Org. Biomol. Chem. 1, pp. 240-243, 2003.

21. P. Furuta, J. Brooks, M. E. Thompson and J. M. J. Frechet, "Simultaneous light emission from a mixture of dendrimer encapsulated chromophores: A model for single-layer multichromophoric organic light-emitting diodes", J. Am. Chem. Soc. 125, pp. 13165-13172, 2003.

22. N. Nishiyama, H. R. Stapert, G. D. Zhang, D. Takasu, D. L. Jiang, T. Nagano, T. Aida and K. Kataoka, "Lightharvesting ionic dendrimer porphyrins as new photosensitizers for photodynamic therapy", Bioconjug. Chem. 14, pp. 58-66, 2003.

23. G. Juzeliūnas and D. L. Andrews, in Resonance Energy Transfer, eds D. L. Andrews and A. A. Demidov, Wiley, Chichester, pp. 65-107, 1999.

24. B. Wieb van der Meer, in Resonance Energy Transfer, eds D. L. Andrews and A. A. Demidov, Wiley, Chichester, pp. 151-172, 1999.

25. D. L. Andrews and A. M. Bittner, "Energy-Transfer in a Static Electric-Field", J. Lumin. 55, pp. 231-242, 1993.

26. G. J. Daniels and D. L. Andrews, "The electronic influence of a third body on resonance energy transfer", J. Chem. Phys. 117, pp. 6882-6893, 2002.

27. K. Becker and J. M. Lupton, "Efficient light harvesting in dye-endcapped conjugated polymers probed by single molecule spectroscopy", J. Am. Chem. Soc. 128, pp. 6468-6479, 2006.

28. P. Allcock, R. D. Jenkins and D. L. Andrews, "Laser-assisted resonance-energy transfer", Phys. Rev. A 6102, $023812,2000$.

29. J. Chen, M. A. Reed, A. M. Rawlett and J. M. Tour, "Large on-off ratios and negative differential resistance in a molecular electronic device", Science 286, pp. 1550-1552, 1999. 
30. Z. Q. Yang, N. D. Lang and M. Di Ventra, "Effects of geometry and doping on the operation of molecular transistors", Appl. Phys. Lett. 82, pp. 1938-1940, 2003.

31. E. M. Just and M. R. Wasielewski, "Picosecond molecular switch based on the influence of photogenerated electric fields on optical charge transfer transitions", Superlattice Microst. 28, pp. 317-328, 2000.

32. M. Alvaro, M. N. Chretien, B. Ferrer, V. Fornes, H. Garcia and J. C. Scaiano, "First molecular switch encapsulated within the cavities of a zeolite. A dramatic lifetime increase of the charge-separated state", Chem. Commun., pp. 2106-2107, 2001.

33. B. S. Ham, “A novel method of all-optical switching: Quantum router", Etri. J. 23, pp. 106-110, 2001.

34. S. Sangu, K. Kobayashi, A. Shojiguchi, T. Kawazoe and M. Ohtsu, "Excitation energy transfer and population dynamics in a quantum dot system induced by optical near-field interaction", J. Appl. Phys. 93, pp. 2937-2945, 2003.

35. O. Wada, "Femtosecond all-optical devices for ultrafast communication and signal processing", New J. Phys. 6, 183, 2004.

36. J. Zhang, Y. Fu and J. R. Lakowicz, "Enhanced Förster resonance energy transfer (FRET) on a single metal particle", J. Phys. Chem. C 111, pp. 50-56, 2007.

37. J. Zhang, Y. Fu, M. H. Chowdhury and J. R. Lakowicz, "Enhanced Förster resonance energy transfer (FRET) on a single metal particle. 2. Dependence on donor-acceptor separation distance, particle size, and distance from metal surface”, J. Phys. Chem. C 111, pp. 11784-11792, 2007.

38. D. L. Andrews and R. G. Crisp, "Optically activated energy transfer: array implementation”, J. Opt. A 8, pp. S106-S112, 2006.

39. D. S. Bradshaw and D. L. Andrews, "Optical control and switching of excitation transfer in nano-arrays", in Nonlinear Frequency Generation and Conversion: Materials, Devices, and Applications VII, ed. Peter E. Powers, Proc. SPIE 6875, 68750O, 2008.

40. D. S. Bradshaw and D. L. Andrews, "Optically controlled resonance energy transfer: Mechanism and configuration for all-optical switching", J. Chem. Phys. 128 (in press). 